\title{
A Practical Integration of Automatic Generation Control and Demand Response
}

\author{
Dylan J. Shiltz and Anuradha M. Annaswamy
}

\begin{abstract}
For a power grid to operate properly, electrical frequency must be continuously maintained close to its nominal value. Increasing penetration of distributed generation, such as solar and wind generation, introduces fluctuations in active power while also reducing the natural inertial response of the electricity grid, creating reliability concerns. While frequency regulation has traditionally been achieved by controlling generators, the control of Demand Response resources has been recognized in recent smart grid literature as an efficient means for providing additional regulation capability. To this end, several control methodologies have been proposed recently, but various features of these proposals make their practical implementations difficult. In this paper, we propose a new control algorithm that facilitates optimal frequency regulation through direct control of both generators and Demand Response, while addressing several issues that prevent practical implementation of other proposals. In particular, i) our algorithm is ideal for control over a large, low-bandwidth network as communication and measurement is only required every 2 seconds, ii) it enables Demand Response resources to recover energy lost during system transients, and iii) it accommodates both measured disturbances and unmeasured disturbances. We demonstrate the viability of our approach through dynamic simulations on a 118-bus grid model.
\end{abstract}

Index Terms-Optimal AGC, direct load control, demand response, frequency regulation

\section{INTRODUCTION}

Maintaining a constant balance between generation and consumption of power is critical to effective power system operations. Several control layers maintain this balance at various time scales. Primary frequency control, based on governor action, is a decentralized control system that adjusts the mechanical power of a generator in response to deviations in local frequency. Following a disturbance, primary control arrests the decline (or rise) in grid frequency in less than a minute or so, but will not restore it to nominal. Secondary control (also called Automatic Generation Control (AGC)) updates generator set-points every few seconds in a centralized fashion in an attempt to restore nominal frequency and/or inter-area tie line flows to their scheduled values [1] [2]. While effective, the current AGC system does not incorporate DR, and it does not in general allocate generation optimally. Finally, tertiary control (also called economic dispatch) calculates optimal system set-points, typically every 5 minutes.

This work was supported by the NSF initiative, Award no. EFRI-1441301. A part of this work was conducted while the first author was a graduate summer intern with the Electric Power Systems Research group at Sandia National Laboratories, Albuquerque, NM.

Both authors are with the Department of Mechanical Engineering, Massachusetts Institute of Technology, Cambridge, MA, 02139 USA e-mail: \{djshiltz,aanna\}@mit.edu
In recent literature, control of DR resources has been proposed as a means to improve many aspects of power system operation, including frequency regulation (see for example [4]-[20], and [4] for a detailed overview). Unlike most generation units, loads can respond to control signals almost instantaneously [5] and the development of advanced metering infrastructure (AMI) [6] has made real-time load control achievable if appropriate control signals are provided. Many control strategies such as [7]-[10] adjust loads based on local frequency, enabling loads to mimic the natural response of synchronous generators. These approaches have been shown to improve the primary frequency response of the grid in both simulations and in small-scale field tests [11]. However, one of the challenges with load control is balancing the objectives of consumers with those of the grid [4], and so the cost (or disutility) to consumers must also be considered.

In [12], an optimal load control (OLC) strategy is developed that minimizes disutility from deferred consumption and grid frequency deviations, and an analytical guarantee of stability for such strategies is provided. In [13], this strategy is expanded to restore nominal frequency following a disturbance. However, these strategies are not integrated with existing primary and secondary control on the generation side. Simultaneous optimal control of generation and deferrable loads using Lagrange multiplier methods was explored in [14][20]. However, there are several issues in these approaches that make their practical implementations difficult.

A common feature to many of the approaches in [4]-[20] is to design and analyze the control system assuming rapid or even instantaneous communication is available, which is not practical for implementation on large networks. As noted in [21], power system SCADA communications and measurements can experience delays of several hundred milliseconds, depending on the communication medium. Such large delays can have a substantial impact on the stability and settling time of network control systems [22]. In this paper, we propose an algorithm for frequency control with DR-compatible loads that is practically implementable. In particular, our algorithm requires discrete communications and measurements only once every 2 seconds (i.e. a time-scale already achieved by existing AGC implementations).

Another issue with load control that is not often addressed is the so-called recovery peak [4] in which loads consume additional power following a period of deferment. Some types of DR, such as electric vehicles, have requirements on how much energy they need to consume over a certain horizon [23]. Other types of DR are shiftable (i.e. their required energy is fixed, but their time of consumption is flexible) [24]. Our 
algorithm addresses these effects by ensuring that DR entities eventually recover all of their energy following a system transient.

Finally, unlike many approaches, our algorithm can accommodate unmeasured disturbances - that is, disturbances that are not directly available to the control law and thus can only be implicitly detected via frequency deviations. This is important for practical implementation in a real power system, in which load fluctuations, line losses, and other disturbances can impact frequency but are difficult to measure directly.

The outline of this paper is as follows. In Section II we model the dynamics of the electric grid and derive a controller to send the grid state to its optimal set-point. In Section III we present the details of our simulations on the IEEE 118-bus test case and discuss the results of these simulations. Section IV provides concluding remarks.

\section{Controller Derivation}

In this section we describe the proposed network-based optimal AGC controller. We start by modeling the dynamics of the power grid, including frequency dynamics of individual buses. Then we design an algorithm that iteratively calculates the optimal generation and consumption set-points. These iterations are broadcast over a wide area network and serve as control references for generators and flexible consumers. Finally, we discuss modifications to enable Demand Response resources to recover their energy following a large transient event (e.g. a rapid drop in renewable generation or a generator tripping offline).

\section{A. Power System Dynamics}

Our dynamic model for the power system is based on [19]. The power grid can be modeled as an undirected graph in which buses are modeled as nodes and transmission lines as edges. As in [19], we assume that a bus is either a generator bus $\mathcal{G}$ with a single synchronous generator or a load bus $\mathcal{L}$ with aggregated flexible power consumption. In addition, every bus has a fixed, conventional consumption. Buses are connected via transmission lines $\mathcal{E}$. An arbitrary connected grid topology may be converted to this form by adding fictitious buses [25]. For simplicity, we adopt a linearized DC power flow model [26] which includes the following assumptions: i) voltage magnitudes of buses remain fixed, ii) resistances of electrical lines are negligible, iii) voltage angle differences are small, iv) reactive power flows are neglected. To simplify notation, we define these state variables as their deviation from a nominal set-point, which is solved on a slower time scale (via the real-time market for example). The power network dynamics, at the fast primary level time-scale, can then be written as

$$
\begin{array}{r}
M_{i} \dot{\omega}_{i}+D_{i} \omega_{i}=P_{M_{i}}-P_{D c_{i}} \\
-\Delta_{i}-\sum_{(i, j) \in \mathcal{E}} B_{i j} \delta_{i j}, i \in \mathcal{G}
\end{array}
$$

$$
\begin{gathered}
D_{i} \omega_{i}=-P_{D r_{i}}^{k}-P_{D c_{i}}-\Delta_{i}-\sum_{(i, j) \in \mathcal{E}} B_{i j} \delta_{i j}, i \in \mathcal{L} \\
\dot{\delta}_{i j}=\omega_{i}-\omega_{j},(i, j) \in \mathcal{E} \\
\dot{P}_{M_{i}}=\tau_{i}^{-1}\left(P_{G_{i}}^{k}-P_{M_{i}}-R_{i}^{-1} \omega_{i}\right), i \in \mathcal{G} .
\end{gathered}
$$

In the above equations, $M_{i}$ is generator inertia, $D_{i}$ is the damping rate of a bus, $P_{M_{i}}$ is mechanical power generation, and $P_{D r_{i}}$ is flexible power consumption. Each bus also has conventional (i.e. non-flexible) consumption $P_{D c_{i}}$ and experiences disturbances $\Delta_{i}$. The difference between these quantities is that $P_{D c_{i}}$ is a measured quantity which is directly available to the control algorithm, while $\Delta_{i}$ is an unmeasured disturbance whose effects can only be detected indirectly through system frequency. In Eq. (4) we model the turbinegovernor dynamics as a first order system with time constant $\tau_{i}$ and primary control coefficient $R_{i}$. Finally, we define $\delta_{i j}=\delta_{i}-\delta_{j}=-\delta_{j i},(i, j) \in \mathcal{E}$ as the voltage angle difference between two adjacent buses and $B_{i j}$ as the susceptance of a transmission line $(i, j) \in \mathcal{E}$.

The terms $P_{G_{i}}^{k}$ and $P_{D r_{i}}^{k}$ are setpoints for generators and flexible consumers. These setpoints are updated periodically at discrete time increments $t^{k}, k \in \mathbb{Z}^{+}$every $\Delta t$ seconds and held constant between updates. In the next subsections we discuss how these setpoints are determined, as well as the period $\Delta t$.

To simplify further analysis, we write the power grid dynamics (1)-(4) compactly as

$$
\dot{y}=A_{\text {grid }} y+B_{\text {grid }} v
$$

where $y=\left[\begin{array}{lll}\omega_{G}^{T} & \delta^{T} & P_{M}^{T}\end{array}\right]^{T}, \omega_{G}$ is a set of $\omega_{i}, i \in \mathcal{G}$, $v=\left[\begin{array}{lll}\left(P_{D c}+\Delta\right)^{T} & P_{D r}^{T} & P_{G}^{T}\end{array}\right]^{T}$, and $A_{\text {grid }}$ and $B_{\text {grid }}$ are constant matrices containing parameters of system (1)-(4).

\section{B. Optimal Power Flow}

For our controller to operate efficiently, it is desired to update $P_{G}^{k}$ and $P_{D r}^{k}$ iteratively at each $t^{k}$, such that the controlled system satisfies Optimal Power Flow (OPF) at equilibrium. The purpose of OPF is to determine the most costeffective way to meet power demands, subject to constraints on generators, consumers, and transmission lines. In this paper, we use the DC-OPF formulation given in [17], which includes flexible consumption as decision variables. An independent system operator (ISO) attempts to solve this problem by maximizing Social Welfare, denoted by $S_{W}$ and defined as

$$
S_{W}=\sum_{i \in \mathcal{L}} U_{D r_{i}}\left(P_{D r_{i}}\right)-\sum_{i \in \mathcal{G}} C_{G_{i}}\left(P_{G_{i}}\right)
$$

where quadratic utility curves of flexible consumers and quadratic cost curves of generators are given in (6) and (7) respectively.

$$
\begin{aligned}
U_{D r_{i}}\left(P_{D r_{i}}\right) & =b_{D r_{i}} P_{D r_{i}}+\frac{c_{D r_{i}}}{2} P_{D r_{i}}^{2} \\
C_{G_{i}}\left(P_{G_{i}}\right) & =b_{G_{i}} P_{G_{i}}+\frac{c_{G_{i}}}{2} P_{G_{i}}^{2}
\end{aligned}
$$


The overall DC-OPF can then be formulated as the following optimization problem, written in simplified matrix-vector notation.

$$
\min -S_{W}
$$

subject to

$$
\begin{gathered}
h(x)=P_{D c}+A_{D r} P_{D r}-A_{G} P_{G}+B_{b u s} \theta+\rho=0 \\
P_{G}^{\min } \leq P_{G} \leq P_{G}^{\max } \\
P_{D r}^{\min } \leq P_{D r} \leq P_{D r}^{\max } \\
-P^{\text {max }} \leq B_{\text {line }} \theta \leq P^{\max }
\end{gathered}
$$

Here our decision vectors are voltage angles $\theta$, flexible consumption $P_{D r}$, and generation $P_{G}$. We refer to the entire decision vector as $x=\left[\theta^{T} P_{D r}^{T} P_{G}^{T}\right]^{T}$. Matrices $B_{\text {bus }}$ and $B_{\text {line }}$ give the net power flow out of a bus and through a transmission line, respectively, when multiplied by $\theta$, and $A_{D r}$ and $A_{G}$ are incidence matrices that map flexible consumers and generators to their respective buses. The equality constraints $h(x)=0$ represent power balance at each bus in the grid. The vector $\rho$ corresponds to feedback control from grid frequency, which will be discussed in Section II-D.

Equations (10)-(12) correspond to inequality constraints, written compactly as $g(x) \leq 0$. The components of $g(x)$ are given by $g_{1}(x)=P_{G}^{\min }-P_{G}, g_{2}(x)=P_{G}-P_{G}^{\max }, g_{3}(x)=$ $P_{D r}^{\min }-P_{D r}, g_{4}(x)=P_{D r}-P_{D r}^{\max }, g_{5}(x)=-P^{\max }-B_{\text {line }} \theta$, and $g_{6}(x)=B_{\text {line }} \theta-P^{\max }$, where $g_{n}(x), n=1, \ldots, 6$ are themselves vectors.

\section{An Iterative Solution of $O P F$}

To solve this optimization problem, first we form its Lagrangian with penalty vectors $\lambda$ and $\mu$ for the equality and inequality constraints, respectively.

$$
\begin{aligned}
& L(x, \lambda, \mu)=-S_{W}(x)+\lambda^{T} h(x)+\mu_{1}^{T} g_{1}(x)+\mu_{2}^{T} g_{2}(x) \\
& \quad+\mu_{3}^{T} g_{3}(x)+\mu_{4}^{T} g_{4}(x)+\mu_{5}^{T} g_{5}(x)+\mu_{6}^{T} g_{6}(x)
\end{aligned}
$$

To simplify notation, we re-write the Lagrangian as

$$
L(x, \lambda, \mu)=f(x, \mu)+\lambda^{T} h(x)
$$

We utilize a solution method similar to the one found in [18], which is a Newton-like primal dual interior point method. We define the Hessian matrix as

$$
H=\nabla_{x x}^{2} f(x, \mu)=\left[\begin{array}{ccc}
0 & 0 & 0 \\
0 & -c_{D r} & 0 \\
0 & 0 & c_{G}
\end{array}\right]
$$

We now construct an augmented Hessian matrix by choosing a positive parameter $\gamma$ such that the following matrix is positive definite

$$
\bar{H}=H+\gamma N N^{T}
$$

where $N=\nabla_{x} h(x)$ is a constant matrix. We then define the update equations for $x$ and $\mu$ as

$$
\begin{gathered}
x^{k+1}=x^{k}-\alpha \bar{H}^{-1} \nabla_{x} L\left(x^{k}, \hat{\lambda}^{k}\right) \\
\mu_{n}^{k+1}=\left[\mu_{n}^{k}+K_{\mu} g_{n}\left(x^{k}\right)\right]^{+}, n=1, \ldots, 6
\end{gathered}
$$

where

$$
\begin{gathered}
\hat{\lambda}^{k}=\left(N^{T} \bar{H}^{-1} N\right)^{-1}\left(h\left(x^{k}\right)-N^{T} \bar{H}^{-1} \nabla_{x} f\left(x^{k}, \mu^{k}\right)\right) \\
\nabla_{x} f\left(x^{k}, \mu^{k}\right)=\left[\begin{array}{c}
B_{\text {line }}^{T}\left(\mu_{6}^{k}-\mu_{5}^{k}\right) \\
-b_{D r}-c_{D r} P_{D r}^{k}+\mu_{4}^{k}-\mu_{3}^{k} \\
b_{G}+c_{G} P_{G}^{k}+\mu_{2}^{k}-\mu_{1}^{k}
\end{array}\right] \\
\nabla_{x} L\left(x^{k}, \hat{\lambda}^{k}\right)=\nabla_{x} f\left(x^{k}, \mu^{k}\right)+N \hat{\lambda}^{k}
\end{gathered}
$$

and $\alpha$ and $K_{\mu}$ are positive parameters chosen at the design stage such that iterates (17)-(18) converge. The operation $[\cdot]^{+}=\max (0, \cdot)$. At convergence, the solution satisfies the Karush-Kuhn-Tucker (KKT) optimality conditions which are sufficient for global optimality (see Theorem 1).

\section{Feedback Control using ACE}

One thing to notice about our control law is that the unmeasured disturbances $\Delta_{i}$ in (1) do not appear directly in any of the update equations (17)-(18). As outlined in the Introduction, these disturbances can only be detected through grid frequency.

Large electricity grids are usually divided into balancing areas, which are connected to one another via tie lines. Each balancing area is managed by a corresponding balancing authority, whose goal is to maintain system frequency within acceptable limits. Typically Area Control Error (ACE) is used to determine each balancing authority's obligation to support frequency control by adding or removing generation. Imbalances arise due to discrepancies in supply and demand within the balancing area, as well as discrepancies in tie line flows between areas [1]. In this paper, we include the power imbalance within the balancing area, but neglect discrepancies in tie line flow.

We define the frequency response bias factor of a bus as $\beta_{i}=D_{i}+R_{i}^{-1}, i \in \mathcal{G}$ for generator buses and $\beta_{i}=D_{i}, i \in$ $\mathcal{L}$ for load buses. The total bias of the area is $\beta_{\text {area }}=$ $\sum_{i \in \mathcal{G} \cup \mathcal{L}} \beta_{i}$. Define $\bar{\omega}(t)$ as the average of $\omega_{i}(t), i \in \mathcal{G} \cup \mathcal{L}$. The area control error can be expressed as

$$
A C E(t)=\beta_{\text {area }} \bar{\omega}(t) .
$$

The parameter $\beta_{\text {area }}$ identifies the excess or shortage of power in the grid per unit of frequency deviation. We can equivalently express ACE in terms of the state variables introduced in Eq. (5) as

$$
A C E(t)=\beta_{\text {area }}(Q y(t)+R v(t))
$$

where $Q$ and $R$ are constant matrices of parameters from system (1)-(4) used to calculate $\bar{\omega}(t)$. Typical practice is to measure ACE at discrete time invervals $t^{k}$. To model this 
procedure, we express ACE as a discrete time signal given by

$$
A C E^{k}=\beta_{\text {area }}\left(Q y^{k}+R v^{k}\right)
$$

where $y^{k}$ and $v^{k}$ are discrete samples of continuous variables $y$ and $v$ in (5). We feed the ACE signal into the algorithm through the vector $\rho$ in the power balance constraints (9). Recall that $h\left(x^{k}\right)$ is used to update iterates (17)-(18). We update $\rho^{k}$ as

$$
\rho_{i}^{k+1}=\rho_{i}^{k}-K_{f} \frac{\beta_{i}}{\beta_{\text {area }}} A C E^{k}, i \in \mathcal{G} \cup \mathcal{L}
$$

where $K_{f}$ is a suitably chosen feedback gain (see Theorem 1 for stability analysis). The purpose of $\rho^{k}$ is to distribute power imbalance measured through ACE to individual buses (weighted by their bias factors $\beta_{i}$ ), such that these imbalances can be met optimally by the control algorithm. Unlike existing Automatic Generation Control (AGC), this enables our control algorithm to deploy flexible consumption as well as generation to stabilize grid frequency, and to do so in a way that maximizes Social Welfare.

We note that (25) can be viewed as an aggregation scheme, in an effort to incorporate unmeasured disturbances in generation and load. As ACE is an indirect measure of these disturbances, the use of $\rho$ can be viewed as an aggregate, based on the frequency bias factors of buses. This may lead to suboptimality, which may be reduced by using more advanced algorithms in lieu of (25).

\section{E. Energy Recovery for DR Resources}

Another feature of our control algorithm is the inclusion Demand Response (DR) resources $P_{D r}$ in a way that allows them to recover their energy following a large excursion in system frequency. Our goal is to modify the control algorithm to ensure that the additional net energy consumed or deferred by a DR resource converges to zero. To this end, we introduce a new set of state variables $E_{D r}$ which are scaled measures of the net energy consumed or deferred by the DR resources. This energy is updated as

$$
E_{D r}^{k+1}=E_{D r}^{k}+K_{E} P_{D r}^{k}
$$

where $K_{E}$ is a diagonal matrix of positive scaling values. Some DR resources may need their energy back very quickly, while others can defer for longer periods. The effect of these scaling values on how quickly each DR resource recovers energy is explored in Section III.

We then replace the DR resources' inequality constraints in (18) with

$$
\begin{aligned}
& g_{3}^{\prime}\left(x^{k}\right)=-E_{D r}^{k}-P_{D r}^{k} \\
& g_{4}^{\prime}\left(x^{k}\right)=P_{D r}^{k}+E_{D r}^{k}
\end{aligned}
$$

It is seen that at equilibrium, we have $E_{D r}^{*}=P_{D r}^{*}=0$, which ensures that the total energy consumed converges to zero, and that the power of each DR resource returns to its nominal value.

\section{F. Stability Analysis}

To summarize, the primary and secondary level dynamics of the grid together with our proposed controller can be expressed as the following hybrid dynamic system.

$$
\begin{aligned}
\dot{y} & =A_{\text {grid }} y+B_{\text {grid }} v \\
\mu_{n}^{k+1} & =\left[\mu_{n}^{k}+K_{\mu} g_{n}^{\prime}\left(x^{k}\right)\right]^{+}, n=1, \ldots, 6 \\
x^{k+1} & =x^{k}-\alpha \bar{H}^{-1} \nabla_{x} L\left(x^{k}, \hat{\lambda}^{k}\right) \\
\rho_{i}^{k+1} & =\rho_{i}^{k}-K_{f} \beta_{i}\left(Q y^{k}+R v^{k}\right), i \in \mathcal{G} \cup \mathcal{L} \\
E_{D r}^{k+1} & =E_{D r}^{k}+K_{E} P_{D r}^{k}
\end{aligned}
$$

This includes both the existing primary control system as part of Eq. (29) as well as the new secondary control system given by Eqs. (30)-(33). We analyze stability and convergence properties of this combined primary-secondary system with the following Theorem:

Theorem 1: For properly chosen control parameters $\alpha, K_{\mu}$, $K_{f}$, and $K_{E}$, system (29)-(33) is stable and converges to the global optimum of Problem (8)-(12).

To prove Theorem 1 we make the following assumptions.

Assumption 1: Problem (8)-(12) is feasible. We assume that sufficient generation has been scheduled in advance to meet power demands, and that the transmission system is capable of handling the necessary power flows. This involves a unit commitment problem which is solved at a slower time-scale.

Assumption 2: At equilibrium, a subset of the elements of $\mu$ are projected. As Problem (8)-(12) includes bounds on decision variables, it is not possible for a decision variable to simultaneously equal its minimum and maximum value at equilibrium. Thus, some of the corresponding Lagrange multipliers $\mu$ must be projected.

See Appendix for the proof of Theorem 1.

\section{G. Network Implementation}

Next we discuss how this control algorithm might be implemented over a large network. An independent system operator (ISO) broadcasts set-points $x^{k}$ to each of the buses every $\Delta t$ seconds (in this paper $\Delta t=2$ seconds). Upon receiving their set-points, each generator and DR consumer responds to the ISO with its entry of $\nabla_{x} f\left(x^{k}, \mu^{k}\right)$, given by (20). These quantities can be thought of as the marginal cost or marginal utility for each participant at the current set-point. Each generator and DR consumer is responsible for updating its own value of $\mu$, given by (30), and DR consumers update their own value of $E_{D r}$, given by (33). The ISO is responsible for measuring system frequency, calculating ACE, and updating $\rho$ using (32). At this point, the ISO has everything it needs to compute the next set of set-points $x^{k+1}$, and the process repeats. We note that the existing AGC/SCADA system updates set-points every 4-6 seconds [2], which includes frequency measurement, processing, and communication. Thus, the communication requirements of our control algorithm should be within the capability of existing communication and measurement infrastructure with minimal modification, making the algorithm feasible to implement.

As it is not practical for thousands or millions of devices to interface directly with an ISO or other central authority, 
we envision a hierarchy in which demand response aggregators [4][27][28] communicate with the ISO and distribute control actions for their respective DR resources. These aggregators would gather information from their constituents and determine characteristics of the group (such as the utility curves in Eq. (6)). The design and operation of such aggregators is an open research question and beyond the scope of this paper. Notable work in this area includes [28] which discusses specific modeling methodologies as well as case studies with supermarket HVAC systems.

\section{Simulation Studies ON IEEE 118 Bus GRID}

In this section we simulate the combined primary-secondary control system on a 118 bus grid. We define a measured disturbance as one that can be made available to the control algorithm in real time. These disturbances enter a bus as a change in conventional demand $P_{D c_{i}}$, and is simulated as a known quantity. We define an unmeasured disturbance as one that is not available to the control algorithm, and whose effects are only felt indirectly through the impact on system frequency. These disturbances enter a bus through $\Delta_{i}$ in Eq.'s (1)-(2). Disturbances (both measured and unmeasured) may be used to model a rapid drop in renewable generation, a sharp increase in conventional (inflexible) demand, or a generator tripping offline.

To analyze the performance of the algorithm, we run a total of 3 tests. In Test 1 , we subject the system to an unmeasured disturbance with no feedback control (i.e. $K_{f}=0$ ). This models the primary control response of the system. In Test 2, we subject the system to the same unmeasured disturbance, but this time with feedback control. In Test 3, we subject the system to a measured disturbance with feedback control. In all tests, disturbances have the same magnitude and are distributed randomly among the buses. After 10 seconds of nominal operation, a disturbance of $+5 \%$ of base load is applied, followed by a disturbance of $-3 \%$ of base load 60 seconds later.

To evaluate system performance, we analyze both ACE and the achieved Social Welfare. We define the achieved Social Welfare as

$$
S_{W_{a}}=\sum_{i \in \mathcal{L}} U_{D r_{i}}\left(P_{D r_{i}}\right)-\sum_{i \in \mathcal{G}} C_{G_{i}}\left(P_{M_{i}}\right)
$$

which is identical to (6) but with the commanded generation $P_{G}$ replaced by the actual mechanical generation $P_{M}$.

\section{A. IEEE 118 Bus Grid}

Our test system is a modified IEEE 118-bus test case [29], of which 54 are generator buses and the other 64 are load buses modified with a certain amount of flexible consumption. Each bus also experiences a conventional, fixed demand $P_{D c}$. The system contains a total of 186 transmission lines. Unless otherwise specified, system parameters are taken directly from the test files [29].

For system parameters not given in the test files, we randomly selected values in the following ranges: $M_{i} \in[8,12]$,

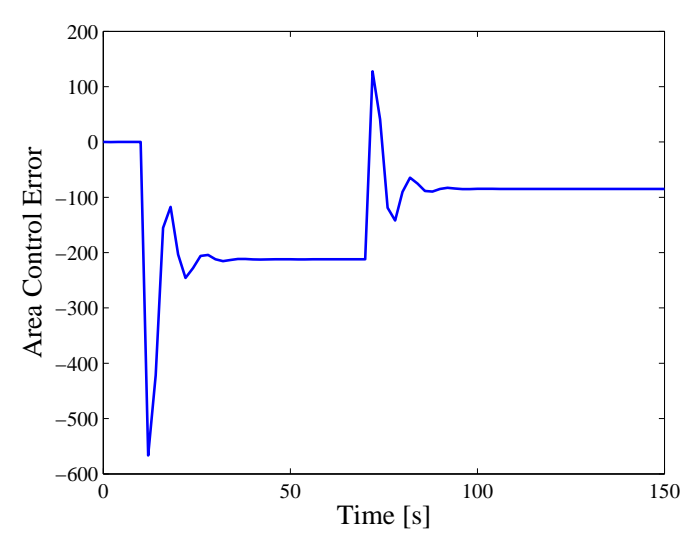

Fig. 1. ACE following an unmeasured disturbance using no feedback

$\tau_{i} \in[3,7], D_{i} \in[0.3,2], R_{i}=0.05, c_{G} \in[0.04,0.05], b_{G} \in$ $[1,2], c_{D r} \in[-2,-1], b_{D r} \in[4,5]$, and $P^{\max }=150 \mathrm{MW}$.

We implement our controller using $\alpha=0.9, K_{\mu}=0.08$, $K_{f}=0.04, K_{E} \in[0.2,0.4]$, and $\gamma=1$. The distribution for $K_{E}$ models potentially different energy recovery requirements of DR resources. As noted previously, we use a communication period $\Delta t$ of 2 seconds.

\section{B. Results and Discussion}

First we discuss Test 1, which models primary control only. As seen in Fig. 1, the ACE stabilizes at negative values, indicating a shortage of power in the system. As this test includes no feedback control, generators adjust based on their physical characteristics rather than cost parameters, and DR resources receive no signal to defer consumption.

Next we discuss Test 2 in which we implement feedback control from ACE through Eq. (32). As seen in Fig. 2, the ACE returns to zero as desired. We see in Figs. 3 and 4 that both generators and flexible consumers are adjusted by the algorithm. The DR resources return to their nominal setpoints at equilibrium, and their total energy consumed converges to zero as shown in Fig. 5. Due to the distribution of gains in $K_{E}$, some of the DR resources recover their energy quickly, while others are allowed a longer recovery period. As the DR resources recover their energy, the generators continue to adjust to maintain ACE at zero. With these improvements, we also see in Fig. 6 that the achieved social welfare is much closer to the optimal value. The slight discrepancy is due to the suboptimality introduced by Eq. (25).

Next we discuss Test 3 in which the disturbance is now measured (i.e. available directly for control). We see in Fig. 7 that the achieved Social Welfare is equal to the optimal value. Plots of active power generation, flexible consumption, and ACE are similar to Test 2 and are not shown.

\section{Summary And CONCluding Remarks}

In this paper we have outlined a practical control algorithm that integrates Automatic Generation Control and Demand Response. This algorithm allows DR resources to participate in frequency control to maximize Social Welfare, enables 


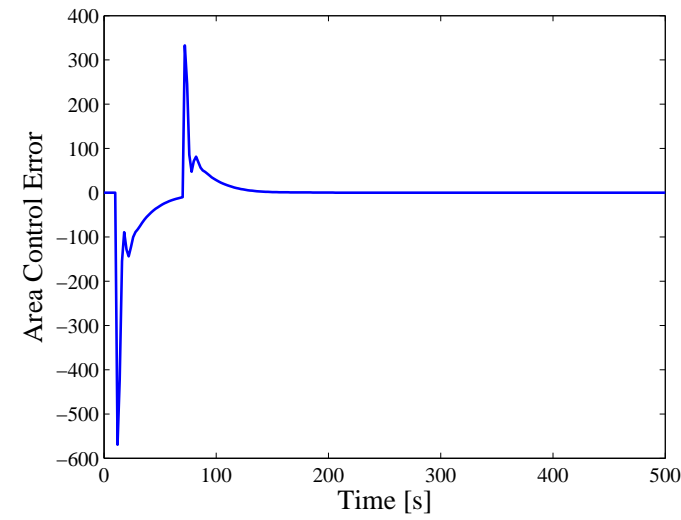

Fig. 2. ACE following an unmeasured disturbance using feedback control

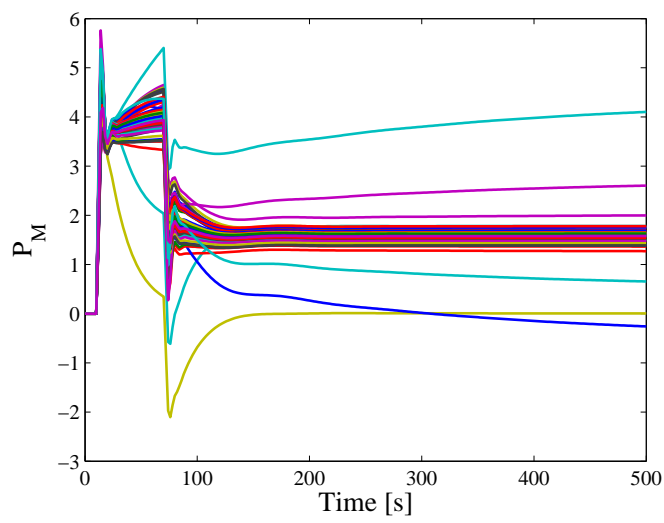

Fig. 3. Generation following an unmeasured disturbance using feedback control

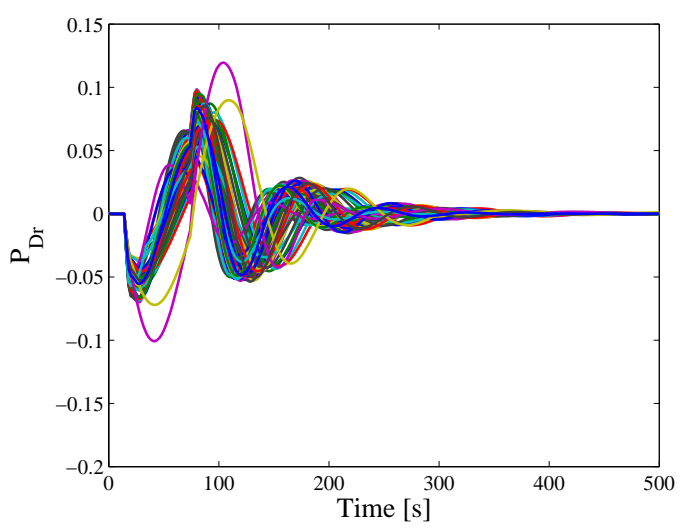

Fig. 4. Demand Response following an unmeasured disturbance using feedback control

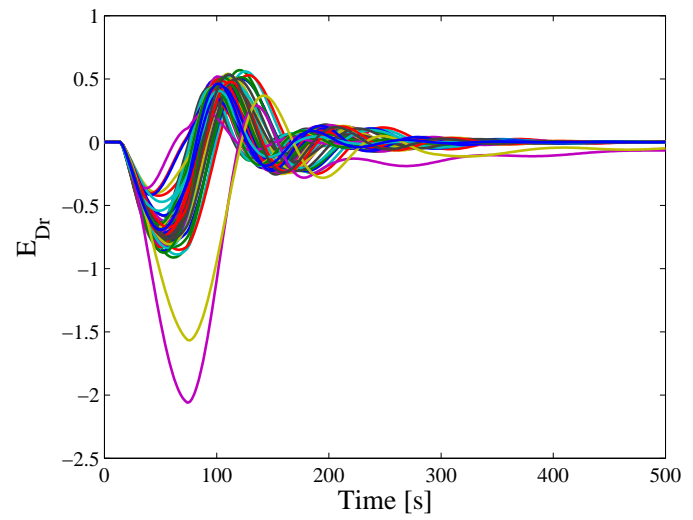

Fig. 5. Net energy consumed by DR resources

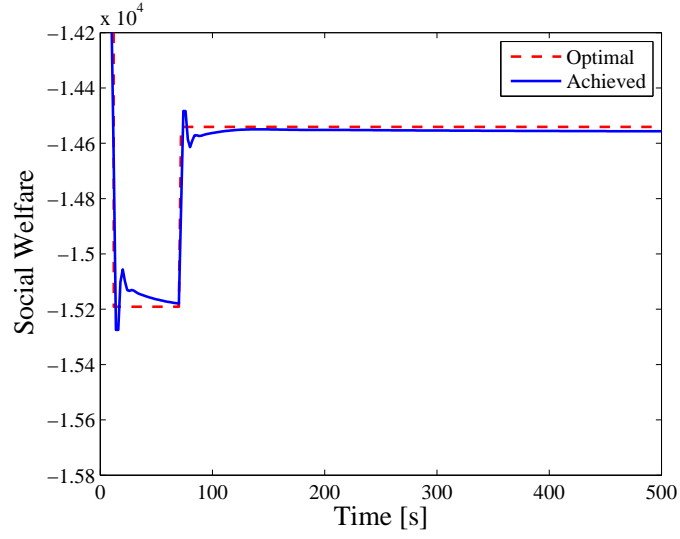

Fig. 6. Social Welfare following an unmeasured disturbance using feedback control

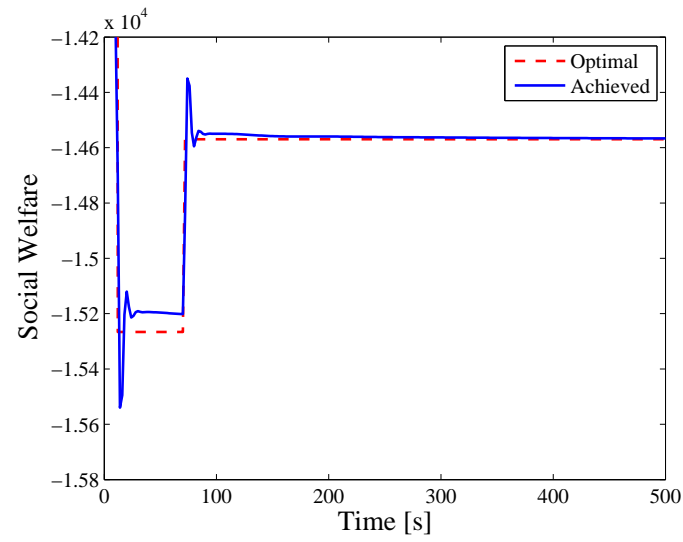

Fig. 7. Social Welfare following a measured disturbance using feedback control 
DR resources to recover their energy following a frequency excursion, accommodates both measured and unmeasured disturbances, and does not introduce excessive communication or measurement requirements. In future work we plan to model larger power systems and subject the model to more detailed disturbance profiles, such as load variation, wind/solar power fluctuation, AC losses, or equipment failures.

\section{REFERENCES}

[1] P. Kundur, Power System Stability and Control, McGraw-Hill, 1997.

[2] North American Electric Reliability Corporation, Balancing and Frequency Control, Technical Report, 2011.

[3] H. Shun-Hsien, D. Maggio, K. McIntyre, V. Betanabhatla, J. Dumas, $\mathrm{J}$. Adams, Impact of wind generation on system operations in the deregulated environment: ERCOT experience, IEEE PES General Meeting, 2009.

[4] D. S. Callaway and I. A. Hiskens, Achieving controllability of electric loads, Proceedings of the IEEE, 99(1), 184-199, 2011.

[5] B. Kirby, Spinning reserve from responsive loads, Oak Ridge Natl. Labs Technical Report ORNL/TM-2003/19, 2003.

[6] C. Woo et al., Now that California has AMI, what can the state do with it?, Energy Policy, 36(4), 2008.

[7] A. Molina-Garcia, F. Bouffard, and D. S. Kirschen, Decentralized demand-side contributions to primary frequency control, IEEE Transactions on Power Systems, 26(1), 411-419, 2011.

[8] M. Donnelly, S. Mattix, D. Trudnowski, J. E. Dagle, Autonomous demand response for primary frequency regulation, Pacific Northwest National Laboratory, Technical Report, 2012.

[9] S. Meyn, P. Barooah, A. Bušić, J. Ehren, Ancillary service to the grid from deferrable loads: the case for intelligent pool pumps in Florida, IEEE Conference on Decision and Control, 52, 2013.

[10] J. Short, D. Infield, F. Freris, Stabilization of grid frequency through dynamic demand control, IEEE Transactions on Power Systems, 22(3), 1284-1293, 2007.

[11] D. J. Hammerstrom et al., Pacific Northwest GridWise Testbed Demonstration Projects, Part II: Grid Friendly Appliance Project, PNNL Technical Report 17079, 2007.

[12] C. Zhao, U. Topcu, N. Li, S. Low, Design and stability of loadside primary frequency control in power systems, IEEE Transactions on Automatic Control, 59(5), 2014.

[13] E. Mallada and S. H. Low, Distributed frequency-preserving optimal load control IFAC World Congress, 5411-5418.

[14] A. Jokic, Price-based optimal control of electrical power systems, $\mathrm{PhD}$ dissertation, Department of Electrical Engineering, Eindhoven University of Technology, 2007.

[15] A. Kiani, A. M. Annaswamy, T. Samad, A Hierarchical Transactive Control Architecture for Renewables Integration in Smart Grids: Analytical modeling and stability, IEEE Tran. on Smart Grid, Special Issue on Control Theory and Technology, 5(4):2054-2065, July 2014.

[16] A. Kiani, A. M. Annaswamy, A Dynamic Mechanism for Wholesale Energy Market: Stability and Robustness, IEEE Tran. on Smart Grid, 5(6):2877-2888, November, 2014.

[17] J. Hansen, J. Knudsen, A. M. Annaswamy, A Dynamic Market Mechanism for Integration of Renewables and Demand Response, IEEE Tran. on Smart Grid, 2015 (to appear).

[18] D. Shiltz, M. Cvetkovic, A. M. Annaswamy, An Integrated Dynamic Market Mechanism for Real-time Markets and Frequency Regulation, IEEE Tran. on Sustainable Energy, 2015 (under review).

[19] X. Zhang, N. Li, and A. Papachristodoulou, Achieving real-time economic dispatch in power networks via a saddle point design approach, IEEE PES General Meeting, 2015.

[20] X. Zhang and A. Papachristodoulou, A real-time control framework for smart power networks: design methodology and stability, Automatica, vol. 58, pp. 43-50, 2015.

[21] B. Naduvathuparambil, M. C. Valenti, and A. Feliachi, Communication Delays in Wide Area Measurement Systems, IEEE Proceedings of the 34th Southeastern Symposium on Systems Theory, pp. 118-122, 2002.

[22] W. Zhang, M. S. Branicky, S. M. Phillips, Stability of Networked Control Systems, IEEE Control Systems, 21(1), pp. 84-99, 2001.

[23] M. K. Petersen, K. Edlund, L. H. Hansen, J. Bendtsen, and J. Stoustrup, A Taxonomy for Modeling Flexibility and a Computationally Efficient Algorithm for Dispatch in Smart Grids, American Controls Conference, 2013.
[24] A. Roscoe and G. Ault, Supporting high penetrations of renewable generation via implementation of real-time electricity pricing and demand response, IET Renewable Power Generation, vol. 4, no. 4, pp. 369-382, 2010.

[25] A. R. Bergen and D. J. Hill, A structure preserving model for power system stability analysis, IEEE Transactions on Power Apparatus and Systems, vol. 100, no. 1, 1981.

[26] M. Ilic, J. Zaborszky, Dynamics and Control of Large Electric Power Systems, John Wiley \& Sons, 2000.

[27] M. Parvania, M. Fotuhi-Firuzabad, M. Shahidehpour, Optimal Demand Response Aggregation in Wholesale Electricity Markets, IEEE Trans. on Smart Grid, 4(4), pp. 1957-1965, 2013.

[28] S. Rahnama, Integration of Large-scale Intelligent Consumers in Smart Grid, PhD dissertation, Department of Electronic Systems, Aalborg University, Denmark, 2015.

[29] Link: https://www.ee.washington.edu/research/pstca/pf118

[30] D. P. Bertsekas, Nonlinear Programming, Athena Scientific, Belmont, Massachusetts, 1999.

[31] K. J. Astrom, B. Wittenmark, Computer-Controlled Systems: Theory and Design, Prentice Hall, 1997.

\section{APPENDIX}

\section{A. Proof of Theorem 1:}

We prove Theorem 1 using the following 3 steps.

Step 1: Problem (8)-(12) is strictly convex. Step 1 follows because the constraints are affine and the objective function is strictly convex over its domain, since the cost curves of the generators are strictly convex and the utility curves of the flexible consumers are strictly concave. This implies that the KKT conditions [30] are sufficient for global optimality.

Step 2: The equilibrium of system (29)-(33) is stable. To show this, we convert power grid dynamics (29) into an equivalent discrete-time model using the methods outlined in [31]. This model is appropriate because the control inputs utilize a zero-order-hold between sampling. Therefore,

$$
y^{k+1}=\Phi y^{k}+\Gamma v^{k}
$$

where $\Phi=e^{A_{\text {grid }} \Delta t}$ and $\Gamma=\int_{0}^{\Delta t} e^{A_{\text {grid }} s} d s B_{\text {grid }}$.

Under Assumption 2, system (29)-(33) is linearized about its equilibrium, with the projected state variables removed. Define $z=\left[\begin{array}{lllll}x^{T} & \mu^{\prime T} & y^{T} & \rho^{T} & E_{D r}^{T}\end{array}\right]^{T}$ and $u^{k}=$ $\left[\begin{array}{cc}P_{D c}^{T} & \Delta^{T}\end{array}\right]^{T}$. The system can be written as

$$
z^{k+1}=A^{\prime} z^{k}+B^{\prime} u^{k}
$$

where

$$
A^{\prime}=\left[\begin{array}{ccccc}
I-\alpha \Omega_{1} & \alpha \Omega_{2}^{\prime} & 0 & \alpha \Omega_{3} & 0 \\
K_{\mu} \Omega_{4}^{\prime} & I & 0 & 0 & K_{\mu} \Omega_{5}^{\prime} \\
\Omega_{6} & 0 & \Phi & 0 & 0 \\
K_{f} \Omega_{7} & 0 & K_{f} \Omega_{8} & I & 0 \\
K_{E} \Omega_{9} & 0 & 0 & 0 & I
\end{array}\right],
$$

where $\Omega_{i}$ are constant matrices that depend on the grid parameters, and $\mu^{\prime}$ is the vector $\mu$ with the projected elements removed. If control gains are chosen such that $A^{\prime}$ is Schur stable, then the equilibrium of (29)-(33) is stable.

Step 3: The equilibrium of (29)-(33) satisfies the KKT conditions. By inspection, Eq. (31) satisfies stationarity of the Lagrangian at equilibrium. Dual feasibility is enforced by the projection operator on $\mu$. From Eq. (30), we observe that if $g_{i}\left(x^{*}\right)<0$ then $\mu_{i}^{*}=0$ and $g_{i}\left(x^{*}\right)>0$ is not possible at equilibrium and so complementary slackness $g_{i}\left(x^{*}\right) \mu_{i}^{*}=0 \forall i$ is satisfied at equilibrium. By combining (19) and (21), we 
observe that $h\left(x^{*}\right)=0$ so primal feasibility is satisfied at equilibrium. Thus, all the KKT conditions are satisfied at equilibrium, which is a sufficient condition for global optimality, proving Theorem 1. 